\title{
PROPERTIES OF DIACYLGLYCEROL KINASE IN ADULT AND FETAL RAT LUNG
}

\author{
HAYAO IDE * and PAUL A. WEINHOLD ** \\ V.A. Medical Center and Department of Biological Chemistry, University of Michigan, Ann Arbor, MI 48105 (U.S.A.)
}

(Received May 11th, 1982)

(Revised manuscript received August 19th, 1982)

Key words: Diacylglycerol kinase; Development; Phosphatidylglycerol; (Rat lung)

Diacylglycerol kinase activity is found in both adult and fetal lung. Approximately 27 and 52\% of the total activity is found in microsomes and cytosol, respectively. The activity is maximal at pH 7.4. The apparent $K_{m}$ for ATP is $0.11 \mathrm{mM}$ and $0.21 \mathrm{mM}$ for cytosol and microsomes, respectively. The apparent $K_{\mathrm{m}}$ for dioleoylglycerol is $0.05 \mathrm{mM}$ for cytosol and $0.14 \mathrm{mM}$ for microsomes. Maximal activity in cytosol and microsomes is obtained with $2.0 \mathrm{mM}$ deoxycholate. Other detergents cannot substitute for deoxycholate. Phosphatidylglycerol stimulates activity in the absence and in the presence of deoxycholate. Phosphatidylserine also stimulates activity, whereas phosphatidylethanolamine was inactive and phosphatidylcholine inhibited the reaction. Linoleic acid produced inhibition. The general properties of the enzyme were similar for fetal and adult lung. Diacylglycerol kinase from microsomes and cytosol fraction from both fetal and adult lung was most active with dioleoylglycerol and diacylglycerol from egg phosphatidylcholine. Significantly lower activity was obtained with dipalmitoylglycerol. Phosphatidylglycerol did not alter the relative substrate preferences. The activity in microsomes increased with development from 19 days gestation to a maximal activity at 21 days gestation. Maximal activity was about 2-fold higher than the adult. The activity dropped rapidly reaching adult values prior to birth (22 days gestation). The activity in cytosol fractions increased gradually from 19 days gestation, reaching adult values by 22 days gestation.

\section{Introduction}

Phosphatidic acid and diacylglycerol are key intermediates in the biosynthetic pathway for glycerolipid synthesis. Both exist at branch points in the pathway. Phosphatidic acid is the precursor for diacylglycerol, subsequently used in the synthesis of phosphatidylcholine, phosphatidylethanolamine, triacylglycerol and for CDPdiacylglycerol which is the precursor for the synthesis of phosphatidylglycerol and phosphatidylinositol. In

\footnotetext{
* Present address: Medical Research Institute, Tokyo Medical and Dental University. 3-10, 2-chome, Kanda-Surugadai, Chiyoda-ku, Tokyo 101, Japan.

** To whom correspondence should be addressed.
}

the usual flow of the pathway, phosphatidic acid is hydrolyzed to diacylglycerol [1]. However, diacylglycerol also can be formed from triacylglycerol via triacylglycerol lipase [2] and may be formed by the reversal of the cholinephosphotransferase-catalyzed reaction [3-5]. Diacylglycerol kinase catalyzes the formation of phosphatidic acid from diacylglycerol [6] and thus may be important in regulating the diacylglycerol concentrations as well as providing an avenue for the conversion of the diacylglycerol formed from phosphatidylcholine, phosphatidylethanolamine and triacylglycerol to phosphatidylinositol and phosphatidylglycerol. Diacylglycerol kinase has been described in several aminal tissues [6-19] and is believed to be involved in a metabolic cycle of 
rapid breakdown and resynthesis of phosphatidylinositol following cell stimulation [19-21]. The present study describes the occurrence and properties of diacylglycerol kinase in adult and fetal rat lung.

\section{Material and Methods}

Adult female and timed pregnant rats were obtained from Holtzman Company, Madison, WI. The age of the fetus was designated by considering the sperm-positive day as day zero. Dioleoylglycerol, dipalmitoylglycerol, diacylglycerol (from egg phosphatidylcholine), phosphatidylglycerol, phosphatidic acid (sodium salt from egg yolk), sodium deoxycholate and ATP were from Sigma Chemical Co. $\left[\gamma-{ }^{32}\right.$ P]ATP $(10-40 \mathrm{Ci} / \mathrm{mmol})$ was from New England Nuclear.

\section{Preparation of lung homogenates and subcellular} fractions

Adult rats were anesthetized with chloral hydrate and the lung perfused with isotonic saline via the pulmonary artery. The lung was ventilated with a small animal respirator throughout the perfusion. The lung was minced and homogenized in $0.25 \mathrm{M}$ sucrose $/ 1.0 \mathrm{mM}$ EDTA $/ 1 \mathrm{mM}$ dithiothreitol $/ 50 \mathrm{mM}$ Tris- $\mathrm{HCl}, \mathrm{pH} 7.4(10 \mathrm{ml}$ per $\mathrm{g}$ lung). The homogenate was centrifuged sequentially at $1000 \times \mathrm{g}$ for $10 \mathrm{~min}$ (cell debris pellet), $20000 \times \mathrm{g}$ for $20 \mathrm{~min}$ (mitochondrial pellet) and $250000 \times \mathrm{g}$ for $60 \mathrm{~min}$ (microsomal pellet and cytosol). The surface of the microsomal pellet was carefully washed three times with homogenizing mixture. The washed pellet was suspended in the $0.25 \mathrm{M}$ sucrose/EDTA/dithiothreitol/Tris mixture. The cytosol and suspended microsomes were stored at $-40^{\circ} \mathrm{C}$.

\section{Preparation of diacylglycerol dispersion}

Two methods were used (1) Diacylglycerol (5.0 $\mu \mathrm{mol}$ ) was added as a chloroform/methanol (1:1) solution to a dry test tube and the solvent was evaporated with a stream of nitrogen. Sodium deoxycholate, $2.0 \mathrm{ml}$ of a $10 \mathrm{mM}$ solution, and 1.0 $\mathrm{ml}$ of water were added and the mixture was sonicated at room temperature four times for 2 min each; (2) diacylglycerol $(5.0 \mu \mathrm{mol})$ and phosphatidylglycerol $(0.8 \mathrm{mg})$ were added in chloro- form/methanol, ( $1: 1)$. The solvent was evaporated with a stream of nitrogen followed by the addition of $2.0 \mathrm{ml}$ of $10 \mathrm{mM}$ sodium deoxycholate and 1.0 $\mathrm{ml}$ water. The mixture was sonicated as in procedure 1 .

\section{Assay of diacylglycerol kinase}

The reaction mixture contained, unless specifically stated otherwise, $100 \mathrm{mM}$ Tris- $\mathrm{HCl}$ buffer pH 7.4, $5 \mathrm{mM} \mathrm{NaF}, 4 \mathrm{mM} \mathrm{MgCl}, 2 \mathrm{mM}$ sodium deoxycholate, $1 \mathrm{mM}\left[\gamma^{32}\right.$ P]ATP $(4000 \mathrm{cpm} / \mathrm{nmol})$, $0.5 \mathrm{mM}$ dioleoylglycerol and $0.15-0.25 \mathrm{mg}$ of enzyme preparation protein in a final volume of 0.5 ml. All ingredients except ATP were incubated for 5 min at $37^{\circ} \mathrm{C}$, after which the reaction was started by the addition of ATP. The reaction mixture was incubated for $10 \mathrm{~min}$ at $37^{\circ} \mathrm{C}$ and stopped by the addition of $3 \mathrm{ml}$ of chloroform/methanol (1:1) containing $0.1 \mathrm{~N} \mathrm{HCl}$. An additional $1.0 \mathrm{ml}$ of chloroform was added and the extract broken into two phases by the addition of $2 \mathrm{ml}$ of $2 \mathrm{M} \mathrm{KCl}$. The chloroform layer was washed four times with methanol $/ 0.02 \mathrm{~N} \mathrm{HCl}(1: 1)$ which was saturated with chloroform. The chloroform was evaporated, the lipid residue dissolved in chloroform/methanol $(1: 1)$ and an aliquot placed into a scintillation vial. Scintillation fluid was added and the amount of radioactivity was determined in a liquid scintillation spectrometer. Product formation was linear with incubation time for $10 \mathrm{~min}$ with adult microsomes and for at least $30 \mathrm{~min}$ for fetal microsomes, fetal supernatant and adult supernatant. The reaction velocity was proportional to the amount of enzyme preparation up to $0.60 \mathrm{mg} \operatorname{protcin} / \mathrm{ml}$.

\section{Chromatographic separation of lipid}

The phospholipids were separated by two-dimensional thin-layer chromatography using silica gel $\mathrm{H}$ plates (Uniplate, Analtech, Newark, DE). The first directional solvent mixture was chloroform/methanol/ammonium hydroxide $(65: 35: 5)$. The plate was dried for $20 \mathrm{~min}$ at $55^{\circ} \mathrm{C}$ in a vacuum oven, adjusted to allow a small flow of air through the oven. The second dimension solvent mixture was chloroform/acetone/methanol/acetic acid/water $(5: 2: 1: 1: 0.5)$. Lipids were visualized with iodine vapor. The lipid-containing areas were scrapped directly into scintillation vials which contained $10 \mathrm{ml}$ of ACS scintillation fluid (New Eng- 
land Nuclear) and the amount of radioactivity was determined.

\section{Results}

Diacylglycerol kinase activity in adult and fetal lung is present in both the microsomal and cytosol fractions ( 27 and $52 \%$, respectively), with relatively low activity in the mitochondrial fraction (3\%). A $\mathrm{pH}$ optimum of 7.4 was observed for both microsomcs and cytosol from adult and fetal lung. The reaction required $\mathrm{Mg}^{2+}$ with maximal activity occurring at $4.0 \mathrm{mM} \mathrm{Mg}{ }^{2+}$. Higher concentrations of $\mathrm{Mg}^{2+}$ produced inhibition. Microsomes from both adult and fetal lung required $10 \mathrm{mM} \mathrm{NaF}$ for maximal activity. The cytosol activity was not changed significantly by $\mathrm{NaF}$. The activities versus ATP concentrations and diacylglycerol concentrations are shown in Figs. 1 and 2, respectively. The specific activity is higher in microsomes than in cytosol. Fetal microsomes have higher specific activities than adult. Double-reciprocol plots of this data were linear. The appartent $K_{\mathrm{m}}$ for ATP was $0.11 \mathrm{mM}$ for cytosol activity and $0.21 \mathrm{mM}$ for microsomes. The apparent $K_{\mathrm{m}}$ for diacylglycerol was $0.05 \mathrm{mM}$ for cytosol preparation and $0.14 \mathrm{mM}$ for microsomes. The apparent $K_{\mathrm{m}}$ values were the same for adult and fetal preparations.

Separation of the products by two-dimensional

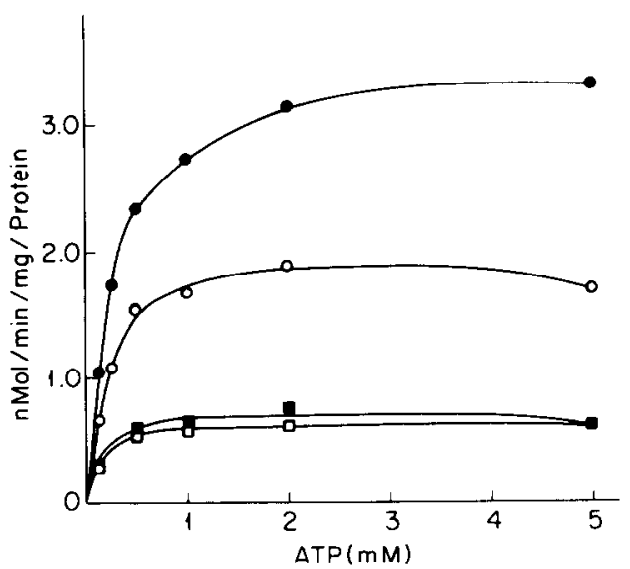

Fig. 1. The effect of ATP concentration on diacylglycerol kinase activity in adult microsomes (O), fetal microsomes (๑), adult cytosol ( $\square$ ) and fetal cytosol ( $\square$ ). Lung from 21 days gestation fetuses was used. $\mathrm{Mg}^{2+}$ was maintained at $4.0 \mathrm{mM}$ throughout.
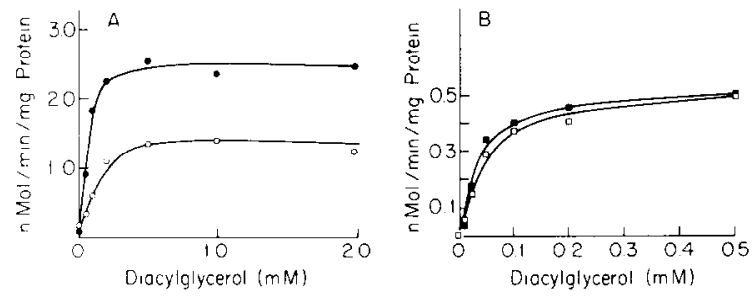

Fig. 2. The effect of diacylglycerol concentration on diacylglycerol kinase activity in adult microsomes ( $O)$, fetal

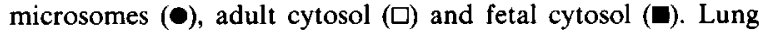
from 21 days gestation fetuses was used. Diacylglycerol was used as substrate. Sodium deoxycholate was maintained at 2.0 $\mathrm{mM}$.

thin-layer chromatography indicated that $97 \%$ of the radioactivity was located in phosphatidic acid and $3 \%$ in lyosphosphatidic acid. No radioactivity was present in other phospholipids.

\section{Effects of detergents and phospholipids on activity}

Diacylglycerol kinase activity in cytosol and microsomes required $2.0 \mathrm{mM}$ sodium deoxycholate for maximal activity (Fig. 3). However, sodium deoxycholate concentrations above $2.0 \mathrm{mM}$ caused inhibition. The non-ionic detergent, Triton X-100, could not replace sodium deoxycholate in the reaction mixture, and inhibited the activity measured in the presence of $2.0 \mathrm{mM}$ sodium deoxycholate. Similar results were obtained with the non-ionic detergent Nonidet P-40. The zwitterionic detergent, sulfobetaine, stimulated activity in the absence of sodium deoxycholate, but the maximal

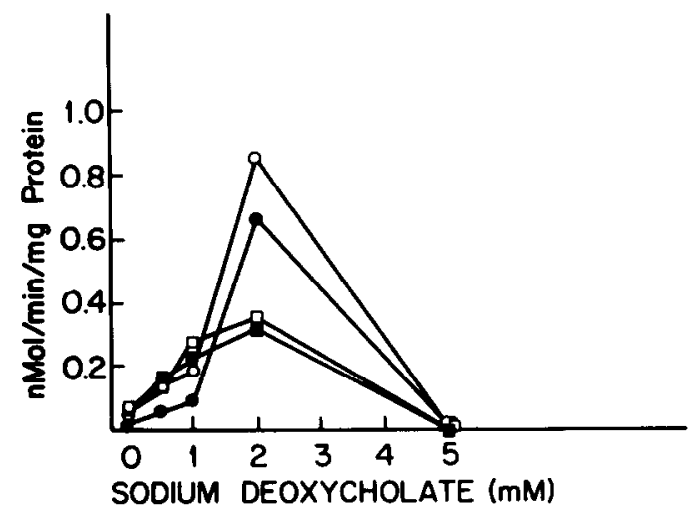

Fig. 3. The effect of sodium deoxycholate on diacylglycerol kinase activity in adult microsomes (๑), fetal microsomes (O), adult cytosol (0) and fetal cytosol (ㅁ) 


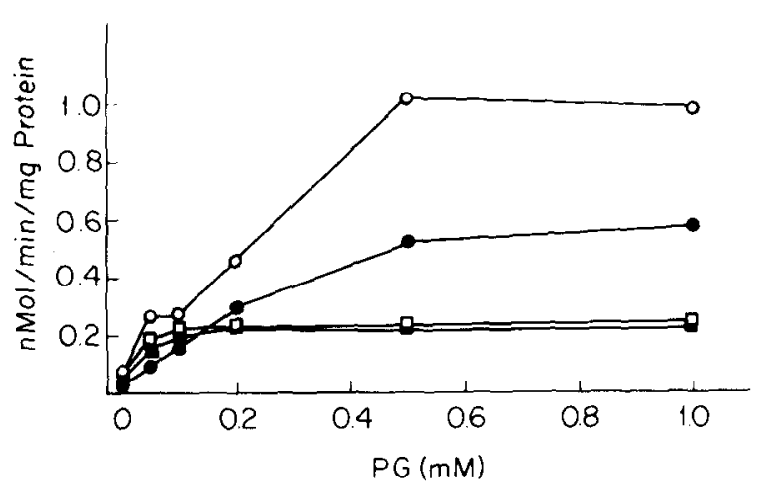

Fig. 4. The effect of phosphatidylglycerol (PG) on diacylglycerol kinase activity in adult microsomes (๑), fetal microsomes (O), adult cytosol $(\square)$ and fetal cytosol $(\square)$ in the absence of deoxycholate.

activity was $50 \%$ of the activity achieved with optimal levels of deoxycholate.

Our previous experience with the assay methods for cholinephosphotransferase indicated that the use of phosphatidylglycerol in the preparation of the diacylglycerol substrate greatly enhanced the activity [22]. The addition of phosphatidylglycerol in the absence of deoxycholate stimulated diacylglycerol kinase activities (Fig. 4). The addition of denxycholate at concentrations below $1.0 \mathrm{mM}$

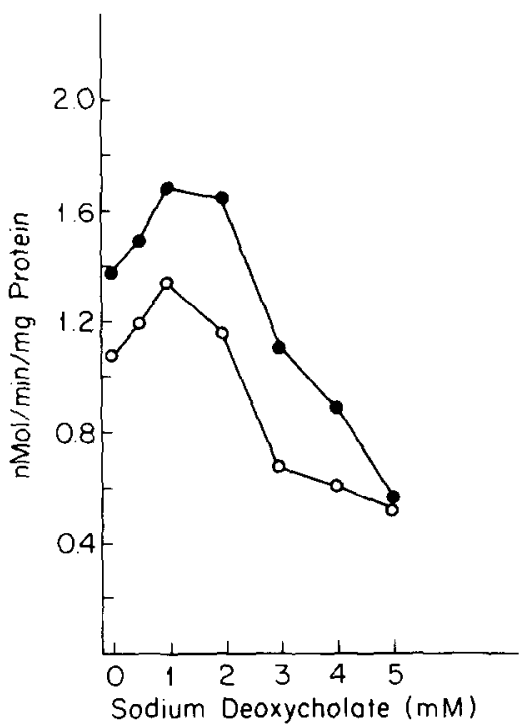

Fig. 5. The effect of sodium deoxycholate on diacylglycerol kinase activity in adult $(O)$ and fetal $(\bullet)$ microsomes in the presence of $0.5 \mathrm{mM}$ phosphatidylglycerol.
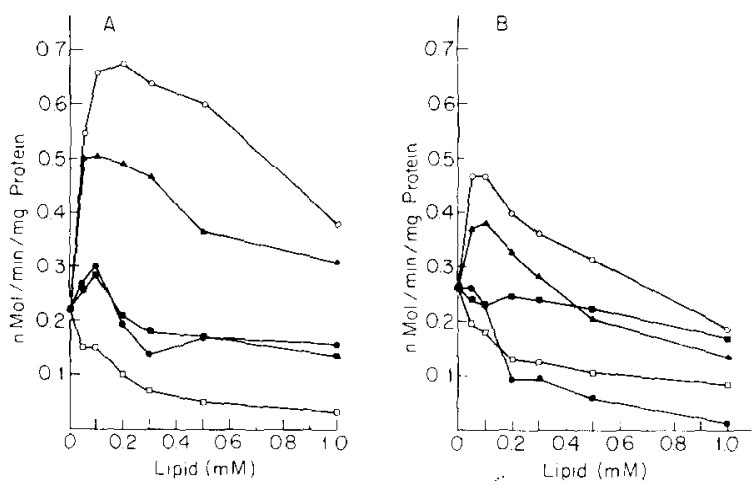

Fig. 6. The effect of phospholipids on diacylglycerol kinase activity in adult cytosol, (A) and in adult microsomes (B). Sodium deoxycholate was maintained at $1.0 \mathrm{mM}$ and dioleoylglycerol was $0.5 \mathrm{mM}$. O. Phosphatidylglycerol; 4 , phosphatidylserine; $\boldsymbol{\square}$, phosphatidylethanolamine; $\square$, phosphatidylcholine; $\bullet$, linoleic acid.

further enhanced the activity produced with optimal concentration of phosphatidylglycerol (Fig. 5). Higher concentrations of deoxycholate gave inhibition. Maximal activity in the presence of phosphatidylglycerol was achieved at $1.0 \mathrm{mM}$ deoxycholate rather than at $2.0 \mathrm{mM}$ deoxycholate, which was observed in the absence of phosphatidylglycerol (Fig. 3). Phosphatidylglycerol, diacylglycerol and sodium deoxycholate were added separately in the experiments reported in Figs. 4 and 5. The sonication of diacylglycerol and phosphatidylglycerol together did not increase the reaction rate beyond that achieved with separate additions.

The relative ability of other phospholipids to stimulate enzyme activity in adult cytosol and microsomes was examined. Phosphatidylglycerol was the most active of those phospholipids studied (Fig. 6). Phosphatidylserine also stimulated the activity in both cytosol and microsomes. Phosphatidylethanolamine was relatively inactive, whereas phosphatidylcholine inhibited the reactions. Linoleic acid inhibited the reaction in both cytosol and microsomes.

\section{Substrate specificity}

Dipalmitoylglycerol was not as good as a substrate for diacylglycerol kinase as was dioleylglycerol or mixed diacylglycerol from egg phosphatidylcholine (Figs. 7 and 8). The addition of 

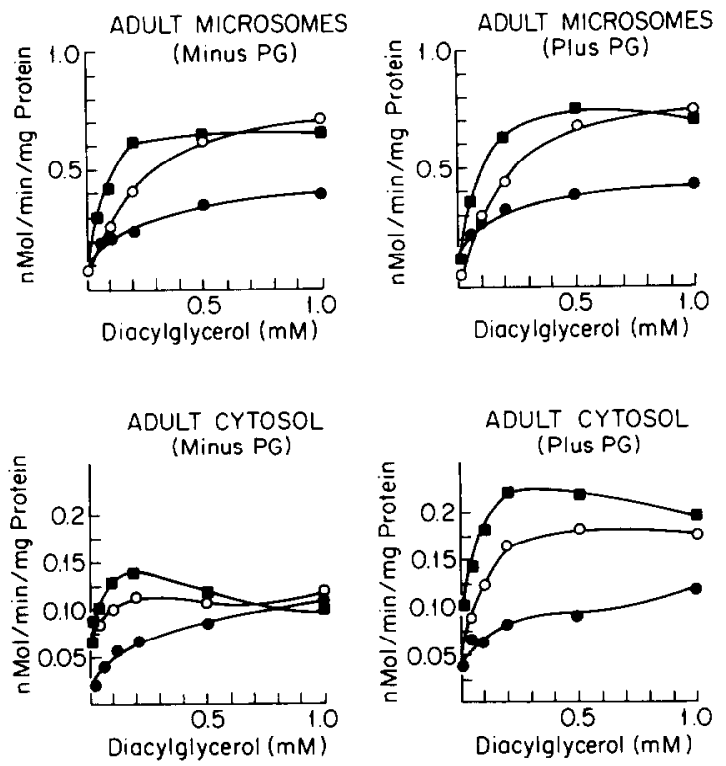

Fig. 7. The diacylglycerol specificity for diacylglycerol kinase in adult microsomes and cytosol. Phosphatidylglycerol (PG), when used, was maintained at $0.1 \mathrm{mM}$. ๑, Dipalmitoylglycerol; $\bigcirc$, dioleoylglycerol; $\mathbf{\square}$, diacylglycerol from egg phosphatidylcholine.
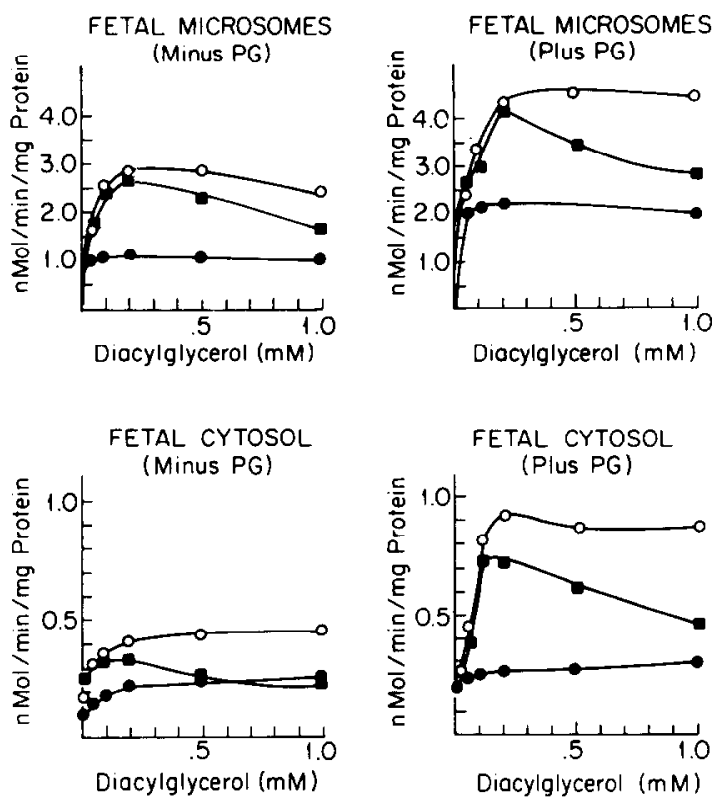

Fig. 8 . The diacylglycerol specificity for diacylglycerol kinase in fetal microsomes and cytosol. Phosphatidylglycerol (PG), when used, was maintained at $0.1 \mathrm{mM}$. Dipalmitoylglycerol; $O$, dioleoylglycerol; $\mathbf{\square}$, diacylglycerol from egg phosphatidylcholine. phosphatidylglycerol to the reaction mixture did not alter the relative substrate preference. The same substrate preference was observed in cytosol and microsomes from both fetal and adult lung. The preference with cytosol preparations was not as striking when the activity was measured without phosphatidylglycerol. The activity in microsomes and cytosol was increased more by phosphatidylglycerol in fetal preparation than in adult preparations.

\section{Activity during development}

The activity in microsomes increased sharply from 19 days gestation to a maximal activity at 21 days gestation (Fig. 9). The microsomal activity dropped to nearly adult levels by 22 days gestation. The activity (units/g lung) in the cytosol fraction increased gradually from 19-days gestation, reaching adult values prior to birth. The developmental profile in microsomes was the same whether expressed per mg protein (A) or per $g$ lung (B), whereas the cytosol profile differed slightly. The activity profile, measured in the presence of phosphatidylglycerol, was essentially the same except that the activity was higher. The
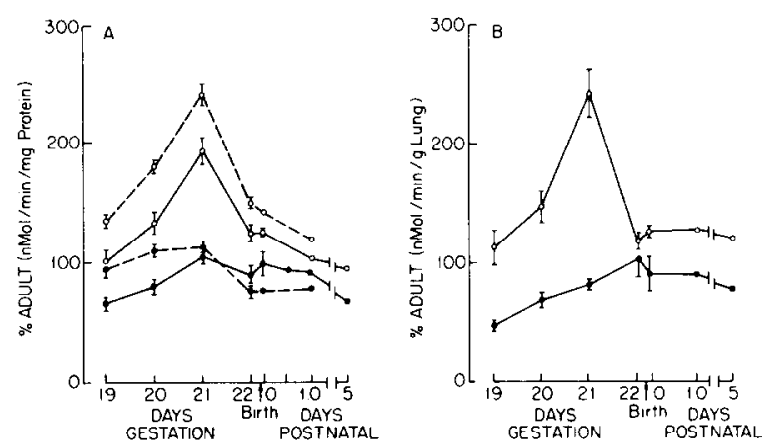

Fig. 9. The activity of diacylglycerol kinase during lung development. The values are averages \pm S.E. The results at 19, 20 and 21 days gestation without added phosphatidylglycerol (- - - ) are averages of activities from five separate litters. The values at 22 days gestation and $3 \mathrm{~h}$ after birth are from three litters. The values with phosphatidylglycerol ( -- ) are averages of three litters. The individual points without standard errors are averages of two litters. The adult values in each case were calculated using activities from adult lung determined simultaneously with the corresponding fetal samples. $\bigcirc$, Microsomes; $\bullet$, cytosol. A, $\mathrm{nmol} / \mathrm{min}$ per $\mathrm{mg}$ protein; $\mathrm{B}, \mathrm{nmol} / \mathrm{min}$ per $\mathrm{g}$ lung. 
increased percent adult values for microsomes is caused by a differential effect of phosphatidylglycerol since the activity in fetal microsomes is stimulated consistantly more than the activity in adult microsomes.

\section{Discussion}

The general properties of diacylglycerol kinase in lung are similar to those reported for other tissues. However, two properties of diacylglycerol kinase in lung appear to differ from those reported for other tissues. Acidic phospholipids, particularly phosphatidylglycerol, stimulate activity in both microsomes and cytosol from lung, whereas Kanoh and Ohno [18] reported that phospholipids did not stimulate the activity in liver cytosol. Secondly, Kanoh and Akesson [13] with liver and Holub and Piekarski [14] with brain observed little substrate specificity towards different species of diacylglycerol. Our results, although not as extensive as reported for liver and brain, suggest that diacylglycerol kinase in lung does not use dipalmitoylglycerol as well as it does diacylglycerols containing unsaturated fatty acids.

We reported previously that the diacylglycerol pool in the lung contains about $30 \%$ disaturated species, most of which is dipalmitoylglycerol [22]. Recent results reported by Tokmakjian and Possmayer [23] also indicated a high amount of disaturated diacylglycerol in rat lung, except their data suggested that a significant proportion of the disaturated species contained stearate. Earlier reports by Okano and co-workers $[24,25]$ and by Moriya and Kanoh [26] indicated that lung diacylglycerol contained $20-25 \%$ disaturated species. While the difference between our results and those of others remains unresolved, the lower preference of diacylglycerol kinase for dipalmitoylglycerol is consistant with a potential role of this enzyme in regulating diacylglycerol species. The diacylglycerol kinase reaction could selectively convert unsaturated diacylglycerol species to phosphatidic acid for the synthesis of phosphatidylinositol and phosphatidylglycerol while conserving the dipalmitoylglycerol needed for the synthesis of dipalmitoylphosphatidylcholine via the cholinephosphotransferase reaction. Since the cholinephosphotransferase reaction in lung ap- pears not to have much specificity for diacylglycerols [22,27], reactions which would preferentially remove unsaturated diacylglycerol, and thus prevent an increase in unsaturated diacylglycerols, may be important in controlling the synthesis of dipalmitoylphosphatidylcholine, particularly under conditions where diacylglycerols are supplied from sources other then the hydrolysis of phosphatidic acid. This sequence of reactions would lead to the formation of unsaturated species of phosphatidylglycerol and phosphatidylinositol. Phosphatidylinositol isolated from rat lung contains predominantly unsaturated molecular species and phosphatidylglycerol, while containing nearly $80 \%$ unsaturated molecular species, also contains relatively high amounts of disaturated species [24]. Furthermore, evidence has accumulated which indicates that unsaturated species of phosphatidylglycerol may be converted to dipalmitoylphosphatidylglycerol by the combined action of phospholipase $\mathrm{A}_{2}$ and palmitoylCoA acyltransferase [28,29]. Thus, unsaturated species of phosphatidylglycerol, which may be produced via the diacylglycerol kinase route, could in turn be converted to disaturated species. Allan et al. [30] suggested previously that diacylglycerol kinase may operate in erythrocyte membranes to control the concentrations of diacylglycerol, since elevated concentrations of diacylglycerol were reported to exert morphological changes in erythrocytes [30] and to promote membrane-fusing $[31,32]$.

The sharp developmental rise in diacylglycerol kinase activity in microsomes between 19 and 21 days gestation is particularly noteworthy since this correlates with the developmental increase in the amount and rate of synthesis of phosphatidylcholine in lung [33]. Most of this increase in phosphatidylcholine is due to an increase in the dipalmitoyl species [34,22]. However, our previous results [22] coupled with recent results by Takmakjian and Possmayer [23] indicated that the diacylglycerol content in fetal lung increased significantly from 19 days gestation to 20 days gestation, after which it remained relatively constant until after birth, at which time it again increased sharply. Thus, diacylglycerol kinase activity begins to increase (19 to 20 days) at a time when the diacylglycerol content is also apparently increasing. This would not be consistent with a strict role 
of diacylglycerol kinase in the regulation of diacylglycerol content. On the other hand, the drop in diacylglycerol kinase activity immediately prior to birth would be consistent with the sharp rise in diacylglycerol content which occurs soon after birth. Thus, if diacylglycerol kinase activity functions at all in the regulation of diacylglycerol content, its role varies with the stage of lung development. The sharp drop in activity between 21 days gestation and birth suggests that the increased fetal activity may he involved with a transitory requirement for the conversion of diacylglycerol to phosphatidic acid. In order for this possibility to make sense in relationship to the increased synthesis of dipalmitoylphosphatidylcholine, which occurs at the same time, a preferential phosphorylation by diacylglycerol kinase of unsaturated species of diacylglycerol over dipalmitoyl species would be necessary. Our in vitro results suggest that the enzyme in 21-day fetal lung does show a preference toward unsaturated diacylglycerols.

Diacylglycerol kinase has been associated with the cycle of phosphatidylinositol breakdown and resynthesis that may occur in tissues exposed to various stimuli $[20,35]$. Conceivably, diacylglycerol kinase also may be associated with phosphatidylinositol metabolism in lung. In the early stages of fetal lung development in the rabbit, lamellar bodies are relatively rich in phosphatidylinositol and contain little phosphatidylglycerol. As development progresses, phosphatidylinositol content drops and phosphatidylglycerol content increases [36]. Although similar studies have not been done in the rat, we observed previously that phosphatidylinositol content of whole lung decreased during the period between 20 days gestation until birth [33]. Furthermore, Okano and Akino [34] reported that phosphatidylglycerol increased in rat lung during that same period of development. Thus, the incrcased activity of diacylglycerol kinase at 20 and 21 days gestation may be involved in the synthesis of phosphatidic acid from diacylglycerol, produced by phospholipase $\mathrm{C}$ hydrolysis of phosphatidylinositol. This would provide a mechanism for the conversion of phosphatidylinositol to phosphatidylglycerol and may be associated with the shift in relative concentration of these two acidic phospholipids. In 1965 we reported the ap- pearance at 21 days gestation of a phospholipid which was highly labeled from ${ }^{32} \mathrm{P}_{\mathrm{i}}$ during slice incubations [33]. At the time, the identity of this phospholipid was unknown. Subsequently, results from other laboratories strongly imply that this phospholipid was phosphatidylglycerol $[37,38]$. The relatively high incorporation of ${ }^{32} \mathrm{P}_{\mathrm{i}}$ into phosphatidylglycerol is consistent with phosphatidylglycerol synthesis via the phosphorylation of diacylglycerol by diacylglycerol kinase. Nevertheless, this suggested flow of diacylglycerol from phosphatidylinositol to phosphatidylglycerol is speculative since it is unknown whether phospholipase C activity towards phosphatidylinositol exists in lung. In addition, the glycerolphosphate phosphatidyltransferase reaction in the synthesis of phosphatidylglycerol appears to be located in mitochondria, at least in adult rat lung [39]. Thus, the phosphatidic acid formed by diacylglycerol kinase in microsomes would not be readily available for phosphatidylglycerol synthesis. However, others have reported reasonable amounts of glycerolphosphate phosphatidyltransferase activity in microsomes [40,41]. Obviously, much more information is needed before the functional significance of diacylglycerol kinase in phospholipid metabolism of the lung can be understood.

\section{Acknowledgements}

This work was supported by the Veterans Administration and by Grant HD02871 from the National Institute of Child Health and Human Devclopment.

\section{References}

1 Van Golde, L.M.G. and Van den Bergh, S.G. (1977) in Lipid Metabolism in Mammals (Snyder, F., ed.), pp. 1-33, Plenum Press, New York

2 Van Golde, L.M.G. and Van den Bergh, S.G. (1977) in Lipid Metabolism in Mammals (Snyder, F., ed.), pp. 35-149, Plenum Press, New York

3 Weiss, S.B., Smith, S.W. and Kennedy, E.P. (1957) J. Biol. Chem. 231, 53-64

4 Sundler, R., Akesson, B. and Nilsson, A. (1974) Biochim. Biophys. Acta 337, 248-254

5 Kanoh, H. and Ohno, K. (1973) Biochim. Biophys. Acta 306, 203-217

6 Hokin, M.R. and Hokin, L.E. (1959) J. Biol. Chem. 234, $1381-1386$ 
7 Hokin, L.E. and Hokin, M.R. (1963) Biochim. Biophys. Acta $67,470-484$

8 Prottrey, C. and Hawthorne, J.N. (1967) Biochem. J. 105, 379-392

9 Lapetina, E.G. and Hawthorne, J.N. (1971) Bichem. J. 122 171-179

10 Call, F.L. and Rubert, M. (1973) J. Lipid Res. 14, 466-474

11 McMurray, W.C. (1975) Can. J. Biochem. 53, 784-785

12 Daleo, G.R., Piras, M.R. (1976) Eur. J. Biochem. 68, 339-346

13 Kanoh, H. and Akesson, B. (1978) Eur. J. Biochem. 85, 225-232

14 Holub, B.J. and Piekarski, J. (1978) J. Neurochem. 31 , 903-908

15 Holub, B.J, and Piekarski, J. (1979) Lipids 14, 308-311

16 Allan, D., Thomas P. and Gatt, S. (1980) Biochem. J. 191, $669-672$

17 Bishop, H.H. and Strickland, K.P. (1980) Lipids 15, 285-291

$18 \mathrm{Kanoh}, \mathrm{H}$. and Ohno, K. (1981) Arch. Biochem. Biophys. 209, 266-275

19 Farese, R.V., Sabir, M.A. and Larson, R.E. (1981) Biochemistry 20,6047-6051

20 Michell, R.H. (1975) Biochim. Biophys. Acta 415, 81-147

21 Lapetina, E.G., Billah, M.M. and Cuatrecasas, P. (1980) in The Regulation of Coagulation (Mann, K.G. and Taylor, E.B., eds.), pp. 491-497, Elsevier North-Holland, Amsterdam

22 Ishidate, K. and Weinhold, P.A. (1981) Biochim. Biophys. Acta 664, 133-147

23 Tokmakjian, S. and Possmayer, F. (1981) Biochim. Biophys. Acta $666,176-180$

24 Okano, G., Kawamoto, T. and Akino, T. (1978) Biochim. Biophys. Acta 528, 385-393
25 Okano, G. and Akino, T. (1979) Lipids 14, 541-546

26 Moriya, T. and Kanoh, H. (1974) Tohoku, J. Exp. Med. $112,241-256$

27 Miller, J.C. and Weinhold, P.A. (1981) J. Biol. Chem. 256, $12662-12665$

28 Batenburg, J.J., Post, M., Oldenborg, V. and Van Golde, L.M.G. (1980) Expt. Lung Res. 1, 57-65

29 Funkhouser, J.D., Batenburg, J.J. and Van Golde, L.M.G. (1981) Biochim. Biophys. Acta 666, 1-6

30 Allan, O., Watts, R. and Michell, R.H. (1976) Biochem. J. $156,225-232$

31 Ahkong, Q.F., Fisher, D., Tampion, W. and Lucy, J.A. (1973) Biochem. J. 136, 147-155

32 Allan, D., Low, M.G., Finean, J.B. and Michell, R.H. (1973) Biocim. Biophys. Acta 413, 309-316

33 Weinhold, P.A., and Villee, C.A. (1965) Biochim. Biophys. Acta 106, 540-550

34 Okano, G. and Akino, T. (1978) Biochim. Biophys. Acta 528, 373-384

35 Michell, R.H. (1979) Trends Biochem. Sci. 4, 128-131

36 Hallman, M. and Gluck, L. (1976) J. Lipid Res. 17, 257-262

37 Rooney, S.A., Canavan, P.M. and Motoyama, E.K. (1974) Biochim. Biophys. Acta 360, 56-67

38 Godinez, R.I., Sanders, S.L. and Longmore, W.J. (1975) Biochemistry, 14, 830-834

39 Mavis, R.D. and Vang, M.J. (1981) Biochim. Biophys. Acta, $664,409-415$

40 Rooney, S.A., Page-Roberts, B.A. and Motoyama, E.K. (1975) J. Lipid Res. 16, 418-425

41 Hallman, M. and Gluck, L. (1975) Biochem. Biophys. Acta, $409,172-191$ 